Rabaska

Revue d'ethnologie de l'Amérique française

\title{
Donatien Laurent (1935-2020) : la Bretagne du coeur aux lèvres
}

\section{Fañch Postic}

Volume 18, 2020

URI : https://id.erudit.org/iderudit/1072921ar

DOI : https://doi.org/10.7202/1072921ar

Aller au sommaire du numéro

Éditeur(s)

Société québécoise d'ethnologie

ISSN

1703-7433 (imprimé)

1916-7350 (numérique)

Découvrir la revue

Citer ce document

Postic, F. (2020). Donatien Laurent (1935-2020) : la Bretagne du coeur aux lèvres. Rabaska, 18, 285-297. https://doi.org/10.7202/1072921ar d'utilisation que vous pouvez consulter en ligne.

https://apropos.erudit.org/fr/usagers/politique-dutilisation/ 


\section{Nécrologie}

\section{Donatien Laurent (1935-2020) ${ }^{1}$ : la Bretagne du cœur aux lèvres}

J'ai fait la connaissance de Donatien Laurent en octobre 1974. Jeune étudiant en lettres classiques, j'étais, pour mon futur mémoire de maîtrise, à la recherche d'un sujet qui allierait deux de mes centres d'intérêt : la Bretagne et la littérature fantastique. Donatien était, m'avait-on assuré, la personne ressource incontournable, à juste titre. Il me reçut dans son bureau du Centre de recherche bretonne et celtique ( $\mathrm{CRBC}$ ) de l'ancienne faculté des lettres de Brest, me conduisit jusqu'à la bibliothèque et, après une brève hésitation, prit sur les rayonnages une collection reliée de $L a$ Paroisse Bretonne de Paris, collection très rare, me dit-il, où se trouvaient publiées les collectes d'un prêtre

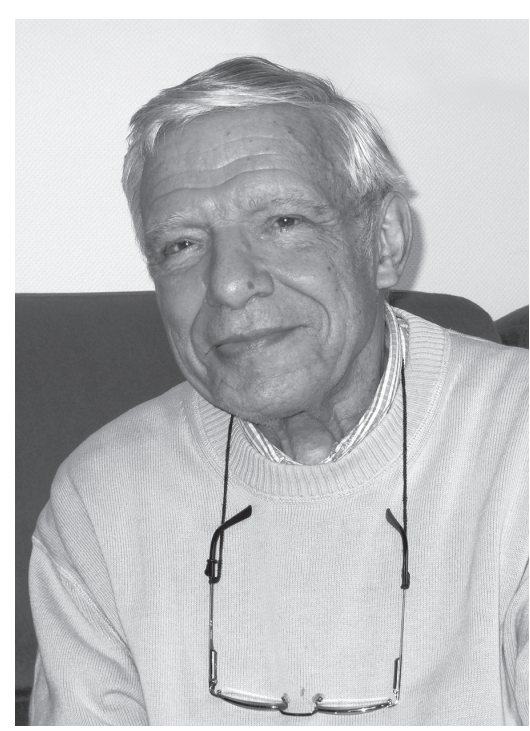

Donatien Laurent

Photo : Jean-Pierre Pichette, 18 octobre 2014 morbihannais, l'abbé François Cadic.

En fait, il venait de recevoir de Marie-Louise Tenèze, grande spécialiste du conte populaire en France, une lettre lui faisant part de la nécessité qu'il y aurait à mettre un peu de clarté dans une collection de contes dans laquelle on se perdait quelque peu. Évidemment, j'étais loin de penser que cette

1. Ce texte reprend en partie la communication que j'avais présentée le 12 décembre 2015 lors de la journée « Retour aux sources » organisée au Quartz à Brest en hommage à Donatien Laurent. Pour une biographie plus détaillée, voir Nathalie Couilloud, « Donatien Laurent - Aux sources de l'ethnologie bretonne et celtique », ArMen, $\mathrm{n}^{\circ}$ 134, mai-juin 2003, p. 16-23, et Michel Treguer, « Chances et génie d'un trépané. Aperçus sur la vie de Donatien Laurent », Fañch Postic (dir.), Bretagnes du cœeur aux lèvres. Mélanges offerts à Donatien Laurent, Rennes, PuR, 2009, p. 7-16. 
double rencontre allait durablement orienter ma vie et tout au long de ces quarante-cinq dernières années je n'ai plus cessé de côtoyer l'abbé Cadic et Donatien Laurent!

\section{Aux sources du Barzaz-Breiz}

Je rencontrais Donatien au moment même où il achevait la rédaction de sa thèse d'État sur les sources du Barzaz-Breiz, le recueil de chants bretons de Théodore Hersart de La Villemarqué qui, publié pour la première fois en 1839, avait été, après sa troisième édition en 1867, l'objet d'un virulente querelle quant à l'authenticité de son contenu. Aujourd'hui, quand il est question de Donatien Laurent... il est inévitablement fait mention du Barzaz-Breiz et réciproquement. En définitive, comme le Barzaz-Breiz a pris toute la place dans la vie de La Villemarqué, le Barzaz-Breiz aura aussi occupé une place prépondérante dans celle de Donatien Laurent. " Le Barzaz-Breiz, c'est sa vie ", titrait d'ailleurs, en page régionale, le quotidien Ouest-France, à l'occasion du colloque consacré à La Villemarqué les 12 et 13 novembre 2015 au manoir de Kernault à Mellac². Mais il convient de préciser que les travaux de Donatien Laurent n'ont pas vraiment pour sujet le Barzaz-Breiz. Le titre précis de sa thèse est d'ailleurs : « La Villemarqué collecteur de chants populaires. Études des sources du premier Barzaz-Breiz à partir des originaux de collecte (1833-1840) ». Donatien Laurent doit même faire une mise au point :

Ma thèse $[\ldots]$ ne portait pas sur le Barzaz-Breiz, ni même à de rares exceptions près, sur la façon dont La Villemarqué s'y est pris pour éditer ses textes, mais sur son activité de collecteur pendant ces années 1833-1838 (et, incidemment, 1840) où il réunissait des documents pour l'ouvrage qu'il avait en projet. [...] Apprécier l'authenticité des chants publiés dans le Barzaz-Breiz serait un tout autre objectif ${ }^{3}$.

\section{Donatien musicien et musicologue}

Le Barzaz-Breiz figure en bonne place dans la bibliothèque familiale des Laurent. Si Donatien est né à Belfort en 1935, où son père est ingénieur chez Alsthom, ce dernier appartient à une famille d'origine cornouaillaise tôt installée à Brest. Sa mère est d'origine nantaise, ce que rappelle le prénom Donatien qui, avec saint Rogatien, est l'un des saints patrons martyrs de l'ancienne capitale du duché de Bretagne. Donatien a 4 ans quand ses parents viennent s'installer à Paris où son père s'investit dans des activités culturelles

2. Nelly Blanchard et Fañch Postic (dir.), Au-delà du Barzaz-Breiz, Théodore Hersart de La Villemarqué (1815-1895), Brest, CRBC, 2016.

3. Donatien Laurent, "Aux origines du «Barzaz-Breiz »: les premières collectes de La Villemarqué (1833-1840)», Bulletin de la Société archéologique du Finistère, tome cI, 1974, p. 173-221. 
et militantes : il sera, à partir de 1947, le président de Ker-Vreiz, foyer culturel breton. Il lui parle bien entendu des chants du Barzaz-Breiz que sa mère, musicienne, chante même en s'accompagnant au piano :

Peu de livres m'ont autant fasciné que le Barzaz-Breiz. Encore étais-je loin d'imaginer que cette rencontre allait peser si durablement sur l'orientation de mon existence... Je me souviens, enfant à Paris, entendre ma mère chanter en s'accompagnant au piano, Baron Joioz, Pennherez Keroulas, Fontanella ..., sur ces mélodies dont la sobre beauté et la plénitude me comblaient ${ }^{4}$.

Donatien Laurent s'intéresse lui-même à la musique - il pratique la cornemuse (écossaise) - et, en 1950, intègre à Paris le Bagad Bleimor. Il y joue alors en couple avec un certain Alan Cochevellou qui se fera connaître quelques années plus tard sous le nom d'Alan Stivell. Avec le bagad, il se rend en Écosse et assiste à l'eisteddfod du pays de Galles. L'Écosse, où il visite le College of Piping de Glasgow, le fascine et, en 1954, il décroche une bourse pour se rendre dans l'île de Skye, aux Hébrides, étudier l'histoire du clan Mac Leod dont les sonneurs héréditaires, les fameux Mac Crimmon, ont créé au XVI ${ }^{\mathrm{e}}$ siècle le premier collège de sonneurs d'Écosse. En 2001, alors que nous nous trouvions dans une crêperie de Lorient avec des collègues participant à l'Université européenne d'été organisée en collaboration avec le Festival interceltique de Lorient, je vois encore le visage stupéfait de nos amis écossais quand Donatien entonna, en gaëlique, la chanson que lui avait chantée en 1954 une habitante de Skye et qu'il n'avait jamais reprise depuis ! En juillet 1956, Donatien Laurent se rend à nouveau en Écosse avec son ami Herri Leon pour suivre l'enseignement du College of Piping de Glasgow qui organise des stages sur l'île de Skye : ils en seront les deux premiers Bretons diplômés. Donatien réalise également, dès cette époque, ses premiers enregistrements et publie des chroniques musicales régulières dans Ar Soner, la revue de Bodadeg ar Sonerion (Assemblée des sonneurs) dont il a intégré la commission technique chargée des concours.

\section{L'année 1957, le début d'une « seconde vie »}

D'abord inscrit en droit pour répondre à la pression familiale, puis à science po, Donatien préfère les cours de philologie celtique d'Édouard Bachellery auxquels il assiste en auditeur libre à l'École pratique des hautes études. Il suit également des cours d'anglais en Sorbonne et engage, sous la direction d'André Martinet, un travail de linguistique générale. Mais le 24 avril 1957, circulant à vélo à La Baule où il est en vacances pour aller poster son travail, il est percuté par un camion, ce qui lui vaut 18 jours de coma. Il rappellera

4. Id., « La Villemarqué et le Barzaz-Breiz. Naissance de la littérature orale », ArMen n ${ }^{\circ}$, décembre 1988, p. 33. 
volontiers comment ce nombre 18 (triwerc' $h$, c'est-à-dire trois fois six) occupe une place particulière dans les chansons bretonnes. Les médecins se montrent pessimistes quant aux séquelles et suggèrent même à ses parents de le marier à une " gentille » bretonne avec laquelle il pourrait, par exemple, ouvrir une crêperie. Voilà Donatien dans ce qu'il aime à appeler "sa seconde vie ", et finalement pas mécontent d'abandonner définitivement les ennuyeuses études auxquelles on le destinait pour profiter de la liberté de se consacrer totalement à ce qui l'intéresse.

1957 est une année charnière : elle lui donne l'occasion de rencontrer en septembre, lors d'un stage des enseignants laïcs d'Ar Falz (« La Faucille »), l'ethnologue Jean-Michel Guilcher qui deviendra son maître et qu'il suivra à Brest après la création en 1969 du CRBC au sein de la toute jeune Université de Brest. La rentrée universitaire de 1957 voit par ailleurs la création à Paris par André Leroi-Gourhan - sommité scientifique dont il convient de préciser qu'il a des attaches avec la Bretagne et qu'il est aussi sonneur ! - d'une licence libre d'ethnologie. Donatien Laurent s'inscrit aussitôt et, après avoir passé quatre certificats, obtient sa licence en octobre 1962 : Linguistique générale, le

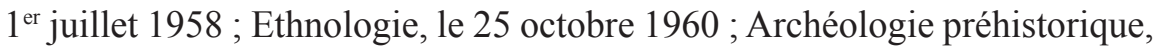
le $1^{\text {er }}$ octobre 1961 ; Histoire des religions, le 29 octobre 1962. Pas si mal pour quelqu'un que la médecine avait déclaré perdu pour les études !

Avec les primes versées par l'assurance, il achète un Nagra qu'il fait modifier à son idée pour, notamment, bénéficier de plus d'autonomie de batterie. Le voilà en possession d'un matériel professionnel qui lui permettra de réaliser des enregistrements de haute qualité. Ce gros travail de collecte mené à une époque où la tradition orale de langue bretonne était encore bien vivante, est, tant par sa qualité que par son ampleur - près de 600 heures d'enregistrement - un véritable trésor patrimonial dont une partie, numérisée et documentée, est désormais accessible en ligne grâce à l'action conjointe du CRBC et de l'association Dastum, et au travail du musicologue Laurent Bigot.

\section{La découverte des manuscrits de La Villemarqué}

Revenons au Barzaz-Breiz. Il est nécessaire de rappeler le cadre tendu dans lequel intervient Donatien Laurent. À la suite de la violente polémique qui, publiquement ouverte en 1867, se réactive après la mort de La Villemarqué en 1895 , ses descendants se refuseront longtemps à ouvrir leurs archives. L'érudit Francis Gourvil, auteur, dès les années 1930, d'articles très critiques sur La Villemarqué, consacre au Barzaz-Breiz une thèse d'université qu'il publie en $1960^{5}$. C'est un remarquable travail de recherche et d'érudition,

5. Francis Gourvil, Théodore-Claude-Henri Hersart de La Villemarqué (1815-1895) et le "Barzaz-Breiz" (1839-1845-1867). Origine-Éditions-Sources-Critiques-Influences, Rennes, Oberthur, 1960. 
mais une thèse à charge qui conclut au caractère largement inventé des chants du Barzaz-Breiz. Pourtant, par les enquêtes que, dès les années 1950, il a menées sur la tradition chantée, Donatien Laurent a acquis la certitude de la part, beaucoup plus grande qu'on ne l'admet à l'époque, des matériaux authentiques dans la composition du Barzaz-Breiz. Et, bien entendu, la réalité des collectes qu'aurait effectuées La Villemarqué et l'existence des cahiers manuscrits sur lesquels il les aurait consignées apparaissent dès lors d'une importance cruciale. Aussi Donatien décide-t-il de «tenter sa chance » (je le cite) et, le 21 juin 1963, écrit au colonel de La Villemarqué et lui expose son projet d'étudier les sources du Barzaz-Breiz. Afin de le rassurer sur l'esprit impartial avec lequel il souhaite engager son travail, à un moment où, après la publication de la thèse de Gourvil, il pourrait légitimement hésiter à ouvrir le fonds d'archives familial, Donatien Laurent lui fait part de la conviction qui est sienne quant à la réalité des sources du Barzaz-Breiz et du poids évident qu'aurait de ce point de vue l'étude des originaux de collecte, s'ils existaient encore. Pierre de La Villemarqué se montre sensible à cette argumentation et, dans sa réponse, exprime à Donatien Laurent le plaisir qu'il aurait à le recevoir ; mais le rendez-vous est manqué pour l'été 1963 et c'est seulement un an plus tard que la rencontre aura finalement lieu : le 14 septembre 1964, Donatien Laurent arrive pour la première fois au manoir de Keransquer, un moment dont il souligne toute la portée émotionnelle :

Le colonel de la Villemarqué me conduisit dans l'ancien manoir de Keransquer dont les bâtiments du XVII ${ }^{\mathrm{e}}$ siècle sont aujourd'hui transformés en communs. Deux petites pièces du premier étage abritaient à cette date les papiers personnels de l'auteur du Barzaz-Breiz et une partie de sa bibliothèque. Ayant ouvert un petit placard mural situé dans l'une d'elles mon hôte commença avec mon aide, à en inventorier le contenu. Tandis que je feuilletais, avec l'émotion que l'on devine, des liasses de lettres et de dossiers, il me montra soudain un petit sac de grosse toile à carreaux sur lequel était collé un papier portant au crayon rouge, presque effacée, la mention « Manuscrits » soulignée d'un double trait, et audessous, ces mots à l'encre noire, d'une main qu'il reconnut être celle de son grand-père, Pierre de la Villemarqué : « Conserver ces manuscrits ».

Le sac contenait trois gros carnets que $M$. de la Villemarqué me tendit en me disant : «Peut-être est-ce là ce que vous cherchez ?»

Il s'agissait bien en effet des fameux textes poétiques dont, après tant d'autres, j'étais en quête. Je les feuilletais rapidement, découvrant au passage, notés en rapides pattes de mouche, des titres prestigieux : Yann eus a Bontorson, Rohan, Merlin ... J'étais déjà comblé de surprises heureuses. Je le fus bien davantage en entendant mon hôte me conseiller d'une voix unie : « Emportez donc tout cela pour l'examiner à loisir. $»^{6}$

6. Donatien Laurent, art. cit., ArMen n ${ }^{\circ}$ 18, 1988, p. 33. 


\section{«Une transcription délicate »}

Voilà donc Donatien en possession des fameux carnets. On aurait pu penser que dès lors l'affaire était classée : il suffisait de les lire, de les transcrire et de les comparer, au besoin, aux textes publiés dans les différentes éditions du Barzaz-Breiz. En fait, le travail ne faisait que commencer. Il est un peu retardé car, en janvier 1964, André Leroi-Gourhan lui propose de participer à la grande enquête pluridisciplinaire de Plozévet où il étudie « Le cycle de la vie » ${ }^{7}$. Il est le seul bretonnant d'une équipe de recherche où figure aussi Françoise Prigent qui deviendra sa femme. En 1965, Donatien Laurent entre au CNRS comme stagiaire et, alors qu'il a un temps envisagé de mener un travail de recherche sur les collectes largement inédites d'Yves Le Diberder, la découverte des carnets de La Villemarqué le décide bientôt d'engager une thèse sur les sources du Barzaz-Breiz, sous la direction d'André Leroi-Gourhan. Parrainé par Georges-Henri Rivière, il rejoint en 1966 le département de littérature orale du Musée national des arts et traditions populaires, dirigé par MarieLouise Tenèze.

La lecture des carnets est en fait d'une très grande difficulté, " une transcription délicate ", écrit pudiquement Donatien en tête de l'un des sous chapitres de sa thèse... C'est peu dire ! Qui a fréquenté les manuscrits de La Villemarqué sait qu'ils sont parfois quasiment illisibles. En fait les carnets sont, comme le note Donatien, des sortes de cahiers de brouillon, contenant des notes griffonnées sous la dictée des chanteurs : " l'écriture en est presque toujours déformée par la hâte. Souvent le contour des lettres devient absolument indiscernable et il ne reste d'un mot qu'un trait plus ou moins onduleux tout juste suffisant, la mémoire et le contexte aidant, pour que le collecteur, revenu à sa table de travail, puisse en retrouver le sens. » Et des points d'interrogations au crayon montrent que parfois La Villemarqué luimême n'arrive pas à se relire! Et puis il y a encore les ratures, les surcharges, les mots recouverts par d'autres mots, quand des notes, des commentaires ne viennent pas se mêler au texte.

Donatien nous livre son secret : « c'est d'abord - cela va de soi - la possession d'une bonne loupe, voire d'un compte-fils, fort utiles dans certains cas complexes. » Il ne lui faudra pas moins de dix ans pour mener à bien la transcription et l'étude du premier des trois carnets : le 16 janvier 1975 à la Sorbonne, il soutient sa thèse d'État devant un jury prestigieux : outre le directeur de thèse, André Leroi-Gourhan, on relève les noms des ethnologues Jean-Michel Guilcher, Robert Creswell et Paul Mercier, de l'historien Jacques Le Goff, du celtisant Édouard Bachellery, du linguiste

7. Id., « Enquête multidisciplinaire de Plozévet. Étude ethno-sociologique. La société traditionnelle, 1. Le cycle de la vie individuelle», s. 1., DGRST-CFrE, s. d. [1966]. 
François Falc'hun. Donatien Laurent obtient la plus haute distinction et les félicitations unanimes du jury.

\section{L'épreuve incontournable du terrain}

En fait, même avec une bonne loupe, il ne faut pas compter arriver au bout de ses peines. Il faut, pour tirer parti des précieux manuscrits, disposer d'une clé bien plus difficile à acquérir : « une solide connaissance du répertoire de la chanson bretonne du XIX ${ }^{\mathrm{e}}$ siècle et de la variété de ses expressions selon les terroirs considérés. » En effet, si la confrontation des versions manuscrites du carnet avec celles recueillies par d'autres collecteurs permet de déchiffrer certains vers illisibles, cela ne saurait suffire. Seule la collecte de terrain permet de comprendre comment La Villemarqué a procédé. Et Donatien, fort de sa propre expérience, a pu, en quelque sorte, se glisser dans la peau de La Villemarqué collecteur, un collecteur pour lequel il ne tarit d'ailleurs pas d'éloges, tant il le trouve doué d'une oreille particulièrement fine qui lui permet de noter exactement ce qu'il entend. Donatien parvient à connaître précisément le mode opératoire de La Villemarqué, à repérer, selon l'écriture ou les couleurs de l'encre, la première notation et les ajouts successifs, les variantes, au gré de nouvelles versions entendues, voire les interventions pour rétablir ce qu'il pense être le texte originel selon les principes d'édition en vigueur à son époque. Il s'attache également à l'aspect linguistique qui lui paraît essentiel, au breton que La Villemarqué utilise dans ses notations, celui de la région natale de Nizon et de Quimperlé.

\section{« Le Maigret breton »}

Ce travail minutieux, souvent proche de l'enquête policière ${ }^{8}$, suppose de constants allers-retours entre la consultation des données manuscrites ou imprimées des collectes déjà disponibles et un terrain encore riche pour ce qui concerne la tradition orale bretonne; il donne lieu à un chapitre important de sa thèse : «Les textes remarquables » où Donatien Laurent analyse les sources de quelques pièces du Barzaz-Breiz, dont certaines étaient unanimement considérées comme des faux, notamment parce qu'elles n'avaient pas d'équivalents dans les autres collectes connues. Or, comme elles sont présentes dans les carnets manuscrits, cela va permettre à Donatien de démontrer que, si La Villemarqué avait pu les retravailler pour leur publication dans le Barzaz-Breiz, elles reposaient bien, à l'origine, sur une collecte. C'est le cas notamment de «Les Chouans », « Merlin Barde », « Le Faucon ». Il étudie aussi d'autres chants qui sont présents dans la tradition populaire, «Le Vassal de Du Guesclin », « Le clerc de Rohan », etc.

8. «DonatienLaurent.Le Maigretbreton»titraitmême le quotidien Le Télégramme le24 mai2016. 
Livrant et analysant les documents inédits des carnets manuscrits, on aurait pu penser que la thèse de Donatien Laurent allait mettre fin à une querelle qui faisait rage depuis déjà un siècle. En fait, Francis Gourvil, après avoir reconnu l'apport déterminant des carnets et du travail de Donatien Laurent, se ravisera. Les positions demeurent tranchées entre ceux qui considèrent le Barzaz-Breiz comme le chef-d'œuvre de la littérature bretonne, à l'image du Kalevala d'Elias Lönnrot, qui, paru en 1835, quelques années avant le Barzaz-Breiz, est devenu le symbole du renouveau identitaire d'une Finlande accédant en 1917 à l'indépendance, et ceux qui voient dans le Barzaz-Breiz une simple mystification qui est, en outre, à l'origine du nationalisme en Bretagne, y compris de ses dérives au moment de la Seconde Guerre mondiale. Dans ce contexte, le point de vue de l'ethnologue a du mal à se faire entendre. L'existence de manuscrits de collecte importe finalement peu.

Le travail que Donatien mène sur les sources du Barzaz-Breiz aboutit à des études de cas qui demeurent encore aujourd'hui des modèles du genre, dont la plus exemplaire est sans aucun doute celle consacrée à la gwerz de Louis le Ravallec.

Bref rappel : le 24 avril 1732, on découvre sur les bords de l'Ellé au Faouët, le corps sans vie de Louis Le Ravallec, un jeune homme de Langonnet, disparu depuis plusieurs jours après s'être rendu au pardon de Saint-Fiacre. Persuadé qu'il s'agit d'un crime, son père utilise toutes les procédures légales à sa disposition, accusant même les juges du Faouët d'être acquis à la cause des assassins et de n'avoir fait qu'une enquête de pure forme. L'affaire est portée en justice mais, au bout de quatre années, se termine par un non-lieu. Les juges concluent à un simple accident : Louis le Ravalec s'est tout simplement noyé ! Par chance toutes les minutes de cette affaire ont été conservées aux archives départementales du Morbihan et constituent un gros dossier dans lequel figurent les dépositions des témoins, les rumeurs, les informations diverses qui ont alors circulé.

En 1965, Donatien Laurent, qui étudie le « Pardon de Saint-Fiacre », l'un des chants du Barzaz-Breiz, est amené à reprendre le dossier. À partir d'un ensemble de versions orales, pour la plupart encore chantées et accompagnées de commentaires, une vérité se fait jour que seule la gwerz composée sur l'affaire permet d'appréhender : il s'agirait en fait d'un drame de la jalousie, au centre duquel se trouve une jeune fille du village de Kerly, Louise Troboul. Or, lors du procès, des témoins avaient cité sa maison comme la dernière où s'était arrêté Louis Le Ravallec avant de reprendre la route de Langonnet. Mais les juges n'avaient jamais pris la peine d'en interroger les habitants. L'étude des registres de baptême, qui démontre l'existence de liens étroits entre la famille Troboul et celles des juges du Faouët, pourrait bien être la clé de leur attitude. 
La gwerz, conclut Donatien Laurent, se pose en gardienne de la mémoire sociale. Elle préserve et transmet $s a$ vérité. La justice humaine peut trancher, en fonction de ses intérêts, de ses solidarités, la parole chantée maintiendra et fera triompher de génération en génération, la force de sa conviction. Contemporaine de l'événement, suscitée par l'émotion populaire et nourrie des rumeurs qui circulent, elle dit l'essentiel de ce qu'elle croit vrai dans une langue simple et imagée, concrète et colorée, mais se garde de toute précision superflue... ou dangereuse. Elle suggère bien plus souvent qu'elle ne décrit et nécessite parfois pour être comprise une information ou des commentaires que tous possèdent sur les lieux-mêmes ou dans le voisinage. Composée et transmise oralement, vivant et se développant en marge de la culture officielle, elle se répand partout où le récit qu'elle livre intéresse et émeut le public, et tant qu'elle conserve pour lui activité et vérité9.

\section{Quand l'oral éclaire l'écrit}

Cas exemplaire qui montre comment une tradition orale peut approcher davantage une vérité que l'écrit des documents d'une procédure, la "gwerz de Louis Le Ravallec » a contribué à faire prendre conscience de l'intérêt historique que peuvent présenter les témoignages oraux. L'article de 60 pages que Donatien Laurent publie en 1967 dans la revue Arts et traditions populaires est un événement. Il encouragera des historiens à prendre désormais en considération les documents oraux dans leurs recherches. Ce sera le cas des fondateurs de la Nouvelle Histoire autour de l'historien Jacques Le Goff, spécialiste du Moyen Âge, qui, - il convient de le rappeler -, participait au jury de thèse de Donatien Laurent.

Il faut évidemment signaler le travail tout aussi remarquable que Donatien Laurent consacre à la Gwerz de Skolan et qui donne lieu à un grand article dans la revue Ethnologie française en $1971^{10}$. La gwerz raconte le retour sur terre de l'âme errante d'un pécheur qui vient demander le pardon de sa mère qui l'avait autrefois maudit pour des crimes qu'il avait commis : il a tué son père, brûlé des églises, tué du bétail, et, ce qui paraît, curieusement, le plus grave, volé un petit livre sacré. Dès le XIX $X^{\mathrm{e}}$ siècle, à la suite de La Villemarqué, on a rapproché cette gwerz d'un poème contenu dans le plus ancien recueil de poésies galloises, le Livre Noir de Carmarthen, un manuscrit du XIII ${ }^{\mathrm{e}}$ siècle. La parenté est évidente : le nom du héros, son apparence et sa monture (duvarc'h, son cheval noir), ses crimes... jusqu'à la métrique. Je renvoie évidement à l'article de Donatien et me contente de souligner un point intéressant de cette affaire : les éléments fournis par l'étude des différentes versions orales

9. Donatien Laurent, « La Gwerz de Louis Le Ravallec », Arts et traditions populaires, ${ }^{\circ} 1$, janvier-mars 1967, p. 19-79. « La Gwerz de Louis Le Ravallec. Enquête sur un crime de 1732 », ArMen nº , février 1987, p. 16-35.

10. Id., «La Gwerz de Skolan et la légende de Merlin», Ethnologie française, ${ }^{\circ} 3-4,1971$, p. 19-54. 
bretonnes ont permis en retour d'éclairer le sens quelque peu énigmatique du texte manuscrit gallois qui présente un curieux dialogue entre un personnage anonyme et un certain Yscolan.

Par le fait même que ce poème est resté vivant en Bretagne et qu'on en recueille encore aujourd'hui plusieurs versions différentes, le récit n'a jamais perdu de sa cohérence : on sait bien qui parle et on dispose de quantité de renseignements complémentaires alors que, dans le texte écrit, au contraire, on est forcé de reconstruire une trame à partir d'éléments extérieurs ${ }^{11}$.

Là encore, l'oral vient utilement éclairer l'écrit.

Parmi les dossiers importants figurent également le siège de Guingamp ou la gwerz du seigneur Nann (« Le roi Renaud ») qui fut le sujet de son intervention - l'une de ses toutes dernières - lors du colloque « La Résistance des marges » à Pointe-de-l'Église en août $2007^{12}$.

\section{La troménie de Locronan et le calendrier celtique}

Restait à Donatien Laurent à achever la transcription et l'étude des carnets 2 et 3 , un travail engagé de longue date qu'il était le seul à pouvoir mener à bien tant ses connaissances approfondies de la façon dont procède La Villemarqué sont irremplaçables. Mais, on le conçoit aisément, après tant d'années passées à transcrire et analyser le contenu du premier carnet de collectes de La Villemarqué et à étudier la transmission du chant populaire, après la soutenance de sa thèse qui le conduit à multiplier articles et conférences, a-t-il sans doute éprouvé un besoin de souffler, voire une forme de lassitude qu'est venue rompre à propos une nouvelle perspective stimulante de recherche : la troménie de Locronan et le calendrier celtique. Je me souviendrai toujours de ce soir de 1978 où, comme souvent, Donatien et sa femme Françoise hébergeaient le jeune étudiant que j'étais alors. Donatien avait déployé sur la table de la salle à manger une très grande carte et entrepris de me faire part, avec l'enthousiasme qui lui était coutumier, de l'intuition qu'il venait d'avoir quant à l'interprétation du parcours sacré de la troménie de Locronan à laquelle il travaillait pour un ouvrage collectif à paraître. Une fois sa démonstration terminée, il me demanda un avis que j'étais évidemment bien incapable de lui donner. Je ne mesurais pas alors qu'en fait d'intuition c'était tout simplement d'une fulgurance dont je venais d'être le témoin : Donatien analysait le pèlerinage millénaire attribué à saint Ronan comme

11. Id., «L'Oral comme objet de recherche en ethnologie », Les Phonothèques entre recherche et culture, numéro spécial de la revue Sonorités, 28-29, 1992, p. 63.

12. Id., "Le "Roi Renaud" en Bretagne ", La Résistance des marges - Exploration, transfert et revitalisation des traditions populaires des francophonies d'Europe et d'Amérique, actes du colloque international organisé du 15 au 18 août 2007 à l'Université Sainte-Anne, Port Acadie, Revue interdisciplinaire en études acadiennes $\mathrm{n}^{\text {os }}$ 13-14-15, printemps 2008-automne 2008-printemps 2009, p. 447-455. 
la transposition dans l'espace d'un calendrier celtique, voire préceltique. Pendant une dizaine d'années, il murit son argumentaire et élargit son étude au calendrier celtique ${ }^{13}$. Cela lui vaut d'avoir aujourd'hui un petit jardin à son nom à deux pas de l'église de Locronan.

\section{La littérature orale aura son Centre de rechceche}

Si la thèse de Donatien Laurent est saluée par le monde scientifique, sa publication ne va pas sans mal. Intéressées, de grandes maisons d'édition parisiennes, dont, m'a-t-il dit, le Mercure de France, renoncent devant l'ampleur de la tâche ; c'est du moins l'argument avancé. Il faudra attendre près de quinze ans pour qu'elle soit enfin éditée par la maison d'édition douarneniste Le Chasse-Marée-ArMen, en $1989^{14}$, année qui marquait le $150^{\mathrm{e}}$ anniversaire de la première édition du Barzaz-Breiz. Les diverses manifestations qui jalonnent l'année 1989 ont un réel retentissement : cela va contribuer à convaincre les élus qui réfléchissent à l'acquisition par le département du Finistère du manoir de Kernault à Mellac, près de Quimperlé, d'en faire un lieu où La Villemarqué et la littérature orale seraient à l'honneur. Louis Le Pensec, ministre et maire de la commune, prend contact avec Donatien. Des réunions sont organisées avec le général de La Villemarqué qui, très vite, donne son accord pour que le fonds d'archives de celui qu'il appelle affectueusement « le barde ", soit l'un des supports du volet scientifique envisagé pour le futur domaine départemental. Dès janvier 1990, un protocole est signé à l'Université de Brest pour la création au domaine de Kernault d'un Centre de recherche et de documentation sur la littérature orale, placé sous la responsabilité de Donatien Laurent, directeur du CRBC, et le CNRS s'engage à ouvrir un poste d'ingénieur d'études chargé d'en assurer la bonne marche. En mars 1990, une association est créée et des réunions se tiennent à Kernault pour réfléchir au programme culturel d'un domaine dont l'achat par le département du Finistère ne sera pourtant effectif que le 12 juin! Ce programme, qui s'appuie sur les travaux du futur Centre de recherche, conduit à l'élaboration d'un plan quinquennal avec, en point d'orgue, 1995 et le centenaire de la mort de La Villemarqué. Retenu au titre des Célébrations nationales par le ministère de la Culture, il donnera lieu à Kernault à une grande exposition « Les Passeurs de mémoires », l'une des premières consacrées à la littérature orale. Beaucoup avaient cherché à

13. Id., « Le Juste milieu. Réflexion sur un rituel de circumambulation millénaire : la troménie de Locronan ", Tradition et histoire dans la culture populaire, rencontres autour de l'œuvre de JeanMichel Guilcher, Grenoble, Centre alpin et rhodanien d'ethnologie, 1990, p. 255-292 ; article repris dans Locronan, la troménie et les peintres (en collaboration avec Fañch Le Henaff et Armel Morgant), Locus Solus, Lopérec, 2013.

14. Id., Aux sources du Barzeiz-Breiz. La mémoire d'un peuple, Douarnenez, Le Chasse-MaréeArMen, 1989, $337 \mathrm{p}$. 
nous en dissuader arguant de l'impossibilité d'exposer une matière orale. Beaucoup aussi nous avaient soutenu l'impossibilité d'organiser à Kernault et à Quimperlé des rencontres scientifiques réunissant des spécialistes de la littérature orale venus du monde entier. Et pourtant ce fut chose faite et les membres de la Conférence sur la ballade ont gardé un souvenir ému de leur congrès de Kernault, tout comme les participants au colloque « La Bretagne et la découverte de la littérature orale en Europe » de Quimperlé. Ces rencontres ont été l'occasion de mesurer l'ampleur et la force du réseau que Donatien Laurent a su construire en Europe et au-delà. On sous-estime trop souvent la renommée qui a pu être la sienne dans les milieux internationaux de la recherche autour de l'oralité.

\section{Pérennité assurée des archives La Villemarqué}

Après son départ à la retraite, les venues de Donatien dans le bureau qu'il avait conservé au CRBC se sont peu à peu espacées. Entre 2014 et 2018, grâce au concours de Marie-Barbara Le Gonidec, son fonds d'archives déposé au CRBC est inventorié et classé. Ces dernières années, ses préoccupations ne portaient pas sur son propre fonds, mais bien sur le devenir des archives de l'auteur du Barzaz-Breiz et de sa bibliothèque, un fonds à l'étude duquel il avait consacré une si large partie de sa vie. Partageant ses inquiétudes, j'avais régulièrement fait part à la famille La Villemarqué de l'intérêt de numériser le fonds avant qu'il ne soit éventuellement vendu, et peut-être dispersé. Au moment d'élaborer la programmation scientifique destinée à marquer, en 2015, le bicentenaire de la naissance de La Villemarqué, Nelly Blanchard et moi avons repris les contacts et obtenu l'accord de la famille pour y intégrer le projet de numérisation des quelque 6000 documents du fonds. Grâce au soutien du Conseil départemental du Finistère, du Conseil régional de Bretagne et de la Ville de Quimperlé, la campagne de numérisation a pu être menée par le CRBC en 2016-2017. Nous avons également aussitôt engagé, grâce au concours de l'équipe du CRBC, un vaste programme de valorisation que nous pensions être le complément scientifique indispensable à la numérisation ${ }^{15}$. Dans ce cadre - à défaut de la publication livresque que Donatien avait initialement envisagée -, la mise en ligne de l'ensemble des trois carnets nous apparaissait primordiale et hautement symbolique. Nelly Blanchard s'est mise au service de Donatien pour lui permettre d'achever un travail si important pour l'histoire littéraire et culturelle de la Bretagne... et si attendu, au point d'avoir alimenté bien des fantasmes !

En novembre 2018, l'ensemble des trois carnets - clichés numériques, transcription et traduction, et présentation - est donc mis en ligne, quelques

15. Cf. www.univ-brest.fr/crbc/menu/Projets_de_recherche/Valorisation + scientifique + des + fichi ers +numériques+du+Fonds+La+Villemarqué. 
jours avant la signature de l'acte de cession par la famille La Villemarqué de son fonds d'archives au Conseil départemental du Finistère. Le 30 novembre 2018, Donatien assiste bien entendu à la cérémonie officielle qui entérine l'entrée du fonds et d'une grande partie de la bibliothèque dans les collections publiques des Archives départementales du Finistère.

\section{Dernières visites}

La pérennité en était désormais assurée pour la plus grande satisfaction de Donatien qui, à chacune de mes visites dans l'établissement brestois où il avait dû se retirer l'été dernier, ne manquait pas d'évoquer tout ce qui lui restait encore à faire ! L'une des dernières, à la fin de 2019, a été pour lui remettre, à la demande de Jean-Pierre Pichette, le dernier numéro de Rabaska : il a pris la revue, m'a parlé du dessin de la couverture qui était le même depuis l'origine, a feuilleté le volume, s'est attardé sur la table des matières, me citant le nom de chaque contributeur qu'il connaissait ou pensait connaître. Puis il m'a pris le bras et son visage s'est éclairé d'un sourire pour me dire combien il était content et même « soulagé » qu'aient été maintenus les liens qu'il avait inaugurés entre la Bretagne et le Canada francophone et qui lui tenaient tant à cœur.

Dans la nuit du 24 au 25 mars, Donatien Laurent s'en est allé paisiblement rejoindre le paradis des Celtes où, je n'en doute pas, sa place était déjà réservée. En 2021, le fonds La Villemarqué sera le support d'une grande exposition proposée à Quimper par le Musée départemental breton et les archives du Finistère en collaboration avec le CRBC : ce sera évidemment l'occasion de rendre à Donatien l'hommage qu'il mérite, lui qui avait, chevillée au corps... et au cœur, une Bretagne largement ouverte sur le monde.

FAÑCh Postic Université de Bretagne occidentale, Brest 